ANL-79-75

(UC-32)

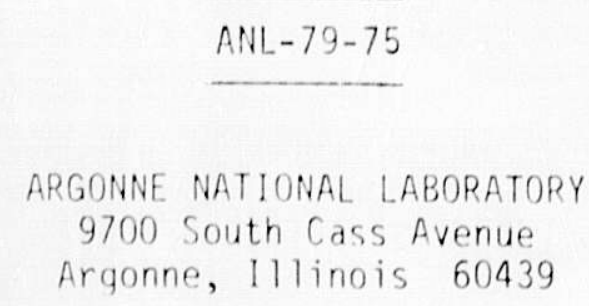

ITERATIVE SOLUTION OF LINEAR PROGRAMS*

by

0. L. Mangasarian ${ }^{\dagger}$

Applied Mathematics Division

July 1979

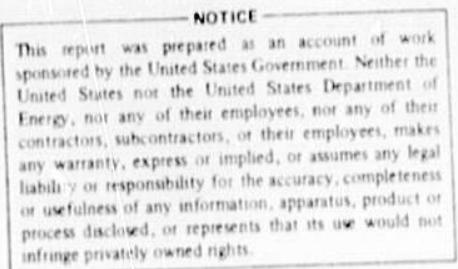

* Research supported by National Science Foundation Grants MCS74-20584 A02 and MCS7901066 and in part by the U.S. Department of Energy.

${ }^{\dagger}$ Current address: Computer Sciences Department and Department of Industrial Engineering, University of Wisconsin, 1210 West Dayton Street, Madison, Wiscons in 53706. 



\section{TABLE OF CONTENTS}

Page

Abstract........................... . . iv

1. Introduction........................... . . . . . 1

2. Iterative Solution of the Quadratic Programming Problem . . . . . . 4

3. Iterative Solution of the Linear Programming Problem . . . . . . . . 8

4. More General Linear Programs . . . . . . . . . . . . . . . . . . . . 11

5. Numerical Results ......................... 14

Acknowledgements . . . . . . . . . . . . . . . . . . . . 19

References.......................... . . . . . . 20 


\title{
ITERATIVE SOLUTION OF LINEAR PROGRAMS
}

by

\author{
0. 1. Mangasarian
}

\section{Abstract}

By perturbing a linear program to a quadratic program, it is possible to solve the latter in its dual variable space by iterative techniques such as successive: over-relaxation (SOR) methods. This provides a solution to the original linear program. 


\section{Introduction}

We shall be concerned here with iterative methods for solving the linear program

$$
\text { Minimize } p^{\top} x \quad \text { subject to } A x \geq b
$$

where $p$ and $b$ are given vectors in $R^{n}$ and $R^{m}$, respectively, and $A$ is a given $m \times n$ real matrix with no rows that are identically zero. The most populal methods for solving this problem are direct pivotal methods such as the simplex method and its variants (Refs。1-2). However, more recently there have been a number of iterative procedures proposed (Refs. 3-8). Some of these (Refs. 3-5) consist of an iterative method for finding a feasible point of the Karush-Kuhn-Tucker inequalities that constitute the optimality conditions of the linear program (1). Others (Refs. 6 and 8) consist of minimizing a nonsmooth reformulated problem or (Ref。7) finding stationary points of an augmented Lagrangian. Our approach here is different. We consider the following quadratic programming perturbation of (1):

$$
\text { Minimize } \frac{\varepsilon}{2} x^{\top} x+p^{\top} x \quad \text { subject to } A x \geqq b
$$

It has been shown (Ref, 9) that (2) has a unique solution $\bar{x}$, for all $\varepsilon$ in $(0, \bar{\varepsilon}]$ for some $\bar{\varepsilon}>0$, which is independent of $\varepsilon$ and which also solves the linear program (1). By working in the dual variable space of (2), we can utilize the iterative techniques developed in (Ref. 10) for solving the symmetric linear complementarity problem to solve (2). It turns out that a sufficient condition 
for the iterative method to lead to a solution is that the constraints of the linear program (1) be stable (Ref. 11), which in this case means that they satisfy the slater constraint qualification (Ref。12)。 It is interesting to note that in order to obtain convergence of the present iterative method, use is made of various recent results concerning linear programs, namely (a) nonlinear perturbation of linear programs (Ref。9) which originated with the uniqueness characterization of linear programming solutions (Ref。13), (b) stability of systems of linear inequalities (Ref。 11) and (c) general sufficient conditions for the convergence of iterative techniques for the solution of the symmetric linear complementarity problem (Ref. 10)。

The outline of the paper is as follows. In Section 2 we describe how our proposed iterative procedure is applied to a general quadratic program with a positive definite Hessian. This procedure may be used also in finding the projection of a point on a polytope. In Section 3 we adapt the procedure of Section 2 specifically for solving the linear program (1) by solving the perturbed quadratic program (2). In Section 4 we give an algorithm for the solution of more general linear programs. In Section 5 we present some numerical results which show that when the perturbation and relaxation parameters are properly chosen our proposed iterative method is competitive with the revised simplex method (Refs. 1-2) and may even be more robust in that it can solve problems for which a revised simplex code fails. We briefly describe now the notation used in this paper. All matrices and vectors are real. For an $m \times n$ matrix $A$, row $i$ is 
denoted by $A_{i}$ and the element in row $i$ and column $j$ by $A_{i j}$. For $x$ in the real $n$-dimensional Euclidean space $R^{n}$, element $j$ is denoted by $x_{j}$. The superscript $T$ denotes the transpose. All vectors are column vectors unless transposed. Superscripts such as $\mathrm{K}^{i}, \mathrm{u}^{i}$ refer to specific matrices and vectors and usually denote iteration numbers. If $u$ is in $R^{m}, u_{+}$denotes the vector in $R^{m}$ with elements

$$
\left(u_{+}\right)_{i}=\max \left\{0, u_{i}\right\}, i=1, \ldots, m
$$

The vector $e$ will denote a vector of ones in $R^{m}$ or $R^{n}$, and $I$ denotes the $m \times m$ identity matrix. The Euclidean norm $\left(x^{\top} x\right)^{\frac{1}{2}}$ of a vector $x$ in $R^{n}$ will be denoted by $\|x\|$. 
2. Iterative Solution of the Quadratic Programming Problem

We sha.1 consider in this section the quadratic program

$$
\text { Minimize } \frac{1}{2} x^{\top} Q x+p^{\top} x \quad \text { subject to } A x \geq b
$$

where $P, b, Q$ and $A$ are respectively a vector in $R^{n}$, a vector in $R^{m}$, a real symmetric positive definite $n \times n$ matrix and a real $m \times n$ matrix. We shall develop an iterative algorithm for solving the dual of (3) (Ref. 12):

$$
\underset{x, u}{\operatorname{Maximize}}-\frac{1}{2} x^{\top} Q x+b^{\top} u \quad \text { subject to } Q x-A^{\top} u+p=0, u \geq 0
$$

which under the positive definite assumption on $Q$ is, upon eliminating $x$, equivalent to

$$
\underset{u \geq 0}{\operatorname{Minimize}} \frac{1}{2} u^{\top} A Q^{-1} A^{\top} u-\left(b+A Q^{-1} p\right)^{\top} u
$$

The proposed iterative procedure will solve (5), and from the solution $u$ the solution $x$ of (3) is then obtained from

$$
x=Q^{-1}\left(A^{\top} u-p\right)
$$

Because the proposed procedure involves the inversion of the matrix Q, it is not, in general, a suitable procedure for solving (3) with a general Q. However, for certain applications such as those requiring the solution of (2), $Q$ is the diagonal matrix $\varepsilon I$, which is easily inverted. Another such problem is that of projecting a point $c$ in $R^{n}$ on the polytope $\{x \mid A x>b\}$ using the Euclidean norm, in which case $Q=I$ and $p=-c$. 
We are now ready to apply the results of Ref. 10 to solve (5) iteratively once we realize that, since $A Q^{-1} A^{\top}$ is positive semidefinite, (5) is equivalent to the following symmetric linear complementarity problem :

$$
v=A Q^{-1} A^{\top} u-\left(b+A Q^{-1} p\right) \geqq 0, u \geqq 0, u^{\top} v=0
$$

We shall use the following special case of Algorithm 2.1 and Remark 2.4 of Ref. 10.

Algorithm 2.1 Let $u^{0}$ be an arbitrary nonnegative vector in $R^{m}$. Having $u^{i}$ compute $u^{i+1}$ as follows:

$$
u^{i+1}=\left(u^{i}-\omega E\left(A Q^{-1} A^{\top} u^{i}-b-A Q^{-1} p+K^{i}\left(u^{i+1}-u^{i}\right)\right)\right)+
$$

where $E$ is a positive diagonal matrix, $K^{i}$ is either the strict lower triangular part $L$ or the strict upper triangular part $U$ of $A Q^{-1} A^{\top}, G$ is the diagonal of $A Q^{-1} A^{\top}$ and

$$
0<\omega<2 / \max _{G_{j j}>0} G_{j j} E_{j j}
$$

Note that for computing purposes $u^{i+1}$ is computed in the order $u_{1}^{i+1}, u_{2}^{i+1}, \ldots . ., u_{m}^{i+1}$ when $k^{i}=L$ and in the reverse of that order when $K^{i}=U$.

The following theorem is a direct consequence of Theorem 2.1 of Ref. 10. 
Theorem 2.1 Let $Q$ be symetric and positive definite. Then, each accumulation point $u$ of the sequence $\left\{u^{i}\right\}$ generated by Algorithm 2.1 soives (5) and the corresponding $x$ determined by $(6)$ is the unique solution of (3).

This theorem does not guarantee the existence of an accumulation of the sequence $\left\{u^{i}\right\}$, whereas the following one does under the slightly more demanding condition of a constraint qualification.

Theorem 2.2 Let Q be symmetric and positive definite, and let the constraints of (3) satisfy the Slater constraint qualification, that is, $A \hat{x}>b$ for some $\hat{x}$ in $R^{n}$. Then the sequence $\left\{u^{i}\right\}$ generated by Algorithm 2.1 is bounded and has at least one accumulation point. Each accumulation point " of $\left\{u^{i}\right\}$ solves (5), and the corresponding $x$ determined by $(6)$ is the unique solution of (3).

Proof Because $A \hat{x}>b$, there exists $a \delta>0$ such that the set $\{x \mid A x>b+\delta c\}$ is nonempty. Let $\tilde{x}$ be the solution of the quadratic program

$$
\text { Minimize } \frac{1}{2} x^{\top} Q x+p^{\top} x \quad \text { subject to } A x \geq b+\delta e
$$

A solution $\tilde{x}$ to this problem exists because $Q$ is positive definite and together with a $\tilde{u}$ in $R^{m}$ satisfies the following Karush-Kuhn-Tucker conditions (Ref. 12):

$$
Q \tilde{x}+p-A^{\top} \tilde{u}=0, \tilde{u} \geqq 0, A \tilde{x} \geqq b+\delta e, \tilde{u}^{\top}(A \tilde{x}-b-\delta e)=0
$$


Hence

$$
\left(A Q^{-1} A^{\top}\right) \tilde{u}-\left(b+A Q^{-1} p\right) \geqq \delta e>0
$$

By Theorem 2.2 and condition (10) of Ref. 10 , the sequence $\left\{u^{\dagger}\right\}$ is bounded and has at least one accumulation point. By Theorem 2.1 above, each accumulation of $\left\{u^{i}\right\}$ solves (5), and the corresponding $x$ determined by $(6)$ is the unique soluticn of (3).

Remark 2.1 The slater constraint qualification is equivalent to the stability condition (Ref. 11) that for each $d$ in $R^{m}$ there exists $(x, c)$ in $R^{m+1}$ satisfying

$$
A x \geq b+\varepsilon d, \varepsilon>0
$$


3. Iterative Solution of the Linear Programing Problem

We now turn our attention back to the linear program (1) and state a result which is a direct consequence of Theorem 1 of Ref.9.

Theorem 3.1 Let the linear program (1) have a solution. Then there exists a real positive number $\bar{\varepsilon}$ such that for each $\varepsilon$ in the interval $(0, \bar{\varepsilon}]$ the unique solution $\bar{x}$ of $(2)$ is independent of $\varepsilon$ and is also a solution of the linear program (1).

From the proof of Theorem 1 of Ref. 9 we can obtain an a posteriori upper bound on $\bar{\varepsilon}$ of the above theorem, namely, $\bar{\varepsilon} \leqq \frac{1}{\bar{\gamma}}$, where $\bar{\gamma}$ is the positive optimal Lagrange multiplier associated with the last constraint of the problem

$$
\text { Minimize } \frac{1}{2} x^{\top} x \quad \text { subject to } A x \geq b, p^{\top} x \leq \bar{\theta}
$$

and where $\bar{\theta}$ is the minimum of problem (1). If $\bar{\gamma}=0$, then $\bar{\varepsilon}$ can be any nonnegative number. There is also another interesting interpretation of $\varepsilon$ (Ref. 14). If we take the dual (Ref. 12) of the convex quadratic program (2), we obtain the problem

$$
\text { Maximize } b^{\top} u-\frac{1}{2 \varepsilon}\left\|A^{\top} u-p\right\|^{2} \quad \text { subject to } u \geq 0
$$

This is precisely the exterior penalty problem assuciated with the dual linear program of (1):

$$
\text { Maximize } b^{\top} u \quad \text { subject to } A^{\top} u=p, u \geq 0
$$

with penalty parameter $\alpha=\frac{1}{\varepsilon}$. Results of ordinary exterior penalty 
methocis (Refo i5) require that $\alpha \rightarrow \infty$ and hence $\varepsilon \rightarrow 0$. However, sharper results that take advantage of 1 inearity of the problem (Ref. 16) require merely that $\alpha \geq \bar{\alpha}$ for some $\bar{\alpha}>0$ or equivalently $\varepsilon \leq \bar{\varepsilon}$ for some $\bar{\varepsilon}>0$. These sharper results correspond to the cited results of (Ref, 9).

We can now combine Theorem 3.1 with Algorithm 2.1 to solve probien (2) with any $\varepsilon$ in $(0, \bar{\varepsilon}]$ and thus obtain a solution to the linear program (1). In particular we set in Algorithm 2.1, $Q=\varepsilon I, E=\varepsilon D^{-1}$, where $D$ is the diagonal of $A A^{\top}$, and obtain the following.

Algorithm 3.1 Choose a positive number $\varepsilon$ and any nonnegative vector $u^{0}$ in $R^{m}$. Having $u^{i}$ compute $u^{i+1}$ as follows :

$$
u^{i+1}=\left(u^{i}-\omega D^{-1}\left(A A^{\top} u^{i}-A p-\varepsilon b+K^{i}\left(u^{i+1}-u^{i}\right)\right)\right)_{+}
$$

where $D$ is the diagonal of $A A^{\top}$, that is,

$$
D_{j j}=A_{j}\left(A_{j}\right)^{\top}, j=1, \ldots, m
$$

$K^{i}$ is either the strict lower triangular part $L$ or the strict upper triangular part $U$ of $A A^{\top}$, and $0<\omega<2$.

Combining Theorems $3.1,2.1$ and 2.2 , we obtain the following two convergence theorems for Algorithm 3.1.

Theorem 3.2 There exists a real positive number $\bar{\varepsilon}$ such that for each $\varepsilon$ in the interval $(0, \bar{\varepsilon}]$, each accumulation point $u$ of the 
sequence $\left\{u^{i}\right\}$ generated by Algorithm 3.1 solves

$$
\underset{u \geq 0}{\operatorname{Minimize}} \frac{1}{2} u^{\top} A A^{\top} u-(\varepsilon b+A p)^{\top} u
$$

and the corresponding $x$ which is independent of $\varepsilon$ and determined by

$$
x=\varepsilon^{-1}\left(A^{\top} u-p\right)
$$

is the unique solution of (2) and is also a solution of the linear program (1).

We again note that Theorem 3.2 does not guarantee the existence of an accumulation point whereas the following theorem does under the additional assumption that the constraints are stable.

Theorem 3.3 Let $A \hat{x}>b$ for some $\hat{x}$ in $R^{n}$. There exists a real positive number $\bar{\varepsilon}$ such that for each $\varepsilon$ in the interval $(0, \bar{\varepsilon}]$, the sequence $\left\{u^{i}\right\}$ generated by Algorithm 3.1 is bounded and has at least one accumulation point. Each accumulation point $u$ of $\left\{u^{i}\right\}$ solves (11), and the corresponding $x$, which is independent of $\varepsilon$ and determined by (12), is the unique solution of (2) and is also a solution of the linear program (1). 


\section{More General Linear. Programs}

We outline in this part of the paper the corresponding results for the case of more general constraints and omit the proofs which are similar to those of Ref. 10 and of Section 3 of this paper. Iri particular we consider here instead of (1) the 1 inear program

$$
\text { Minimize } p^{\top} x \quad \text { subject to } A x \geq b, C x=d
$$

where the additional equality constraint is specified through the $k \times n$ matrix $C$ and the vector $d$ in $R^{k}$. We note that this problem encompasses linear programs of a very general type. We shall again assume, for simplicity, that no rows of $A$ or $C$ are identically

zero and associate with (13) the following quadratic program for some positive $\varepsilon$ :

$$
\text { Minimize } \frac{\varepsilon}{2} x^{\top} x+p^{\top} x \quad \text { subject to } A x \geqq b, C x=d
$$

and the corresponding dual problem

$$
\underset{\substack{\operatorname{Minimize} \\
u \geq 0}}{\ln \left(R^{m+k}\right.} \frac{1}{2}\left(\begin{array}{l}
u \\
v
\end{array}\right)^{\top}\left(\begin{array}{l}
A \\
c
\end{array}\right)\left(A^{\top} C^{\top}\right)\left(\begin{array}{l}
u \\
v
\end{array}\right)-\left(\begin{array}{l}
A p+\varepsilon b \\
C p+\varepsilon d
\end{array}\right)^{\top}\left(\begin{array}{l}
u \\
v
\end{array}\right)
$$

where the relation between $x, u$ and $v$ is given by

$$
x=\varepsilon^{-1}\left(A^{\top} u+C^{\top} v-p\right)
$$

The iterative procedure associated with (15) is as follows. 
Algorithm 4.1 Choose a positive number $\varepsilon$, an arbitrary nonnegative vector $u^{0}$ in $R^{m}$ and an arbitrary vector $v^{0}$ in $R^{k}$. Having $\left(\begin{array}{l}u_{i}^{i} \\ i\end{array}\right)$ compute $\left(\begin{array}{l}u^{i+1} \\ v^{i+1}\end{array}\right)$ as follows

$$
\left.\left(\begin{array}{l}
u_{i+1}^{i+1} \\
v^{i+1}
\end{array}\right)=\left(\left(\begin{array}{l}
u_{i}^{i} \\
v^{i}
\end{array}\right)-\omega D^{-1}\left(\left(\begin{array}{l}
A \\
c
\end{array}\right)\left(A^{\top} c^{\top}\right)\left(\begin{array}{l}
i \\
u_{i}^{i} \\
v^{\prime}
\end{array}\right)-\left(\begin{array}{l}
A p \\
C p
\end{array}\right)-\varepsilon\left(\begin{array}{l}
b \\
d
\end{array}\right)+k^{i}\left(\begin{array}{l}
u^{i+1}-u^{i} \\
v^{i+1}-v^{i}
\end{array}\right)\right)\right)\right)_{\star}
$$

where $D$ is the diagonal of $\left(\begin{array}{l}A \\ C\end{array}\right)\left(A^{\top} C^{\top}\right), K^{i}$ is the strictly lower or upper triangular part of the same matrix, $0<\omega<2$, and

$$
\left(\begin{array}{l}
u \\
v
\end{array}\right)_{\star}=\left(\begin{array}{l}
u_{+} \\
v
\end{array}\right)
$$

Theorem 4.1 There exists a real positive number $\bar{\varepsilon}$ such that for each $\varepsilon$ in the interval $(0, \bar{\varepsilon}]$, each accumulation point $\left(\begin{array}{l}u \\ v\end{array}\right)$ of the sequence $\left\{\left(\begin{array}{l}u^{i} \\ v^{i}\end{array}\right)\right\}$ generated by Algorithm 4.1 solves (15), and the corresponding $x$ determined by (16), which is independent of $\varepsilon$, is the unique solution of (14) and in addition is a solution of the linear program (13)。

Theorem 4.2 If in addition to the assumptions of Theorem 4.1 the constraints of (13) are stable -- that is, there exists an $\hat{x}$ in $R^{n}$ such that $A \hat{x}>b, C \hat{x}=d$, and the rows of $C$ are linearly independent -then for $\varepsilon$ in $(0, \bar{\varepsilon}]$ the sequence $\left\{\left(\begin{array}{l}u_{i}^{i} \\ v\end{array}\right)\right\}$ of Algorithm 4.1 is bounded and hence has at least one accumulation $\left(\begin{array}{l}u \\ v\end{array}\right)$. Each such accumulation point solves (15), and the corresponding $x$, which is 
independent of $\varepsilon$ and determined by (16), is the unique solution of (14) and is also a solution of the linear program (13). 
5. Numerical Results

Some test results were obtained using the iterative SOR Algorithm 3.1 starting with $u^{0}=0$ to solve the linear program (1) on the Argonne National Laboratory IBM 3033 computer running under VM release 5 PLC 11 and the Conversational Monitoring System. For comparative purposes a revised simplex code was also used (Ref. 17). The test pioblems were generated as follows. The matrix A was a fully dense matrix with random elements $A_{i j}$ uniformly distributed in the interval $[-100,400]$. The vectors $b$ and $p$ were chosen such that

$$
b_{i}=\left\langle\begin{array}{l}
\sum_{j=1}^{n} A_{i j} \text { if } \sum_{j=1}^{n} A_{i j}>0 \\
-1+2 \sum_{j=1}^{n} A_{i j} \text { if } \sum_{j=1}^{n} A_{i j} \leq 0 \\
i=1, \ldots, m
\end{array}\right.
$$

and

$$
p_{j}=\sum_{i \in j} A_{i j} \text { where } J=\left\{i \mid \sum_{j=1}^{n} A_{i j}>0\right\}, j=1, \ldots, n
$$

These choices for $b$ and $p$ made the point $x=2 e$ satisfy the constraint qualification $A(2 e)>b$ and the point $x=e$ primal optimal with a minimum value of $\sum_{j=1}^{n} \sum_{i \in J} A_{i j}$. A dual optimal variable is given by $u_{i}=1$ for $i \in J$ and $u_{i}=0$ for $i \notin J$. Results for six cases are summarized in Table 1. Note that for cases 1, 2, 3 and 5 , because $n>m$, the linear program (1) does not have a unique 
solution (Ref. 13). Cases 4 and 6 have a unique solution if and only if the matrix with rows $A_{j}, i \in J$, has linearly independent columns (Ref. 13). Thus the accuracy of the solutions described in Table 1 is measured by (a) the number of figures in agreement between the calculated objective function and the theoretical minimum $\sum_{j=1}^{n} \sum_{i \in j} A_{i j}$, and (b) the $\infty$-norm of the infeasibility of the calculated primal solution $x$, that is, the maximum $\underset{1 \leq i \leq m}{L_{j=1}}\left(b_{i}-A_{i j} x_{j}\right)_{+}$. We now make the following observations regarding Table 1:

(i) Except for cases 4 and 6, the computing times for the two methods are quite similar. For case 6 , the revised simplex method failed, and for case 4 the iterative method took 2.5 times as long as the revised simplex method.

(ii) The revised simplex solutions, when obtained, are more accurate than those of the iterative method.

(iii) The iterative method is more robust in the sense that it never fails to provide some answer when the constraints are stable.

(iv) The values of the perturbation parameter $\varepsilon$ and the relaxation parameter were obtained after some experimenting, but are not necessarily optimal. Table 2 gives a typical range of answers obtained by the iterative method for various values of $\varepsilon$ and $\omega$ which led to the values $\varepsilon=10^{5}$ and $\omega=0.5$ given in Table 1 for the case of $m=250$ and $n=100$. 


\section{TABLE}

Numerical Results for Min $p^{\top} x$ s.t. $A x \geq b$ where $A$ is an $m<n$ matrix

\begin{tabular}{|c|c|c|c|c|c|c|c|c|c|c|c|}
\hline \multirow{3}{*}{ Case } & \multirow{3}{*}{ m } & \multirow{3}{*}{$n$} & \multicolumn{3}{|c|}{ Revised Simplex Method } & \multicolumn{6}{|c|}{ Iterative Method } \\
\hline & & & \multirow{2}{*}{$\begin{array}{c}\text { Seconds of } \\
\text { Virtual } \\
\text { Machine } \\
\text { Time }\end{array}$} & \multicolumn{2}{|c|}{ Accuracy } & \multirow[b]{2}{*}{$\varepsilon$} & \multirow[b]{2}{*}{$\omega$} & \multirow[b]{2}{*}{$\begin{array}{l}\text { No. of } \\
\text { Iterations }\end{array}$} & \multirow{2}{*}{$\begin{array}{c}\text { Seconds of } \\
\text { Virtual } \\
\text { Vachine } \\
\text { Time }\end{array}$} & \multicolumn{2}{|c|}{ Accuracy } \\
\hline & & & & $\begin{array}{l}\text { No. of Correct } \\
\text { Figures in Ob- } \\
\text { jective Function }\end{array}$ & $\begin{array}{l}\infty-i \text { orm of } \\
\text { Primal In- } \\
\text { feasibility }\end{array}$ & & & & & $\begin{array}{l}\text { No. of Correct } \\
\text { Figures in Ob- } \\
\text { jective Function }\end{array}$ & $\begin{array}{l}\text { - Nom of } \\
\text { Primal In- } \\
\text { feasibility }\end{array}$ \\
\hline 1 & 10 & 100 & $<2$ & 15 & $0.324 \times 10^{-11}$ & $10^{3}$ & 0.8 & 136 & $<2$ & 14 & $0.374 \times 10^{-10}$ \\
\hline 2 & 50 & 200 & 6 & 14 & $0.902 \times 10^{-10}$ & $10^{4}$ & 0.8 & 862 & $<2$ & 9 & $0.365 \times 10^{-4}$ \\
\hline 3 & 50 & 850 & 20 & 14 & 0 & $10^{5}$ & 0.1 & 642 & 20 & 12 & $0.257 \times 10^{-5}$ \\
\hline 4 & 100 & 98 & 28 & 15 & $0.218 \times 10^{-9}$ & $10^{5}$ & 0.5 & 1300 & 70 & 4 & $0.400 \times 10^{-5}$ \\
\hline 5 & 100 & 850 & 94 & 16 & $0.144 \times 10^{-8}$ & $10^{6}$ & 0.7 & 915 & 106 & 13 & $0.969 \times 10^{-7}$ \\
\hline 6 & 250 & 100 & 700 & $\begin{array}{l}\text { Failed: Prob } \\
\text { Declared Unb }\end{array}$ & $\begin{array}{l}\text { em } \\
\text { bunded }\end{array}$ & $10^{5}$ & 0.5 & 1114 & 130 & 10 & $0.484 \times 10^{-6}$ \\
\hline
\end{tabular}


TABLE 2

Numerical Results for Iterative Method for Min $p^{\top} \times$ s.t. Ax $>b, m=250, n=100$

\begin{tabular}{|c|c|c|c|c|c|}
\hline \multirow[b]{2}{*}{$\varepsilon$} & \multirow[b]{2}{*}{ (u) } & \multirow{2}{*}{$\begin{array}{l}\text { No, of } \\
\text { Iterations }\end{array}$} & \multirow{2}{*}{$\begin{array}{c}\text { Seconds of Virtual } \\
\text { Machine Time }\end{array}$} & \multicolumn{2}{|c|}{ Accuracy } \\
\hline & & & & $\begin{array}{l}\text { No. of Figures in } \\
\text { Objective Function }\end{array}$ & $\begin{array}{l}\infty \text {-Norm of Primal } \\
\text { Infeasibility }\end{array}$ \\
\hline $10^{4}$ & 0.5 & 500 & 62 & 5 & $0.860 \times 10^{-2}$ \\
\hline $10^{4}$ & 0.2 & 500 & 64 & 4 & $0.196 \times 10^{-1}$ \\
\hline $10^{4}$ & 0.8 & 500 & 62 & 4 & $0.436 \times 10^{-1}$ \\
\hline $10^{5}$ & 0.5 & 500 & 64 & 5 & $0.725 \times 10^{-2}$ \\
\hline $10^{6}$ & 0.5 & 500 & 64 & 4 & 0.164 \\
\hline $10^{5}$ & 0.2 & 500 & 64 & 4 & 0.116 \\
\hline $10^{5}$ & 0.8 & 500 & 62 & 4 & $0.859 \times 10^{-1}$ \\
\hline $10^{5}$ & 0.5 & 1114 & 130 & 10 & $0.484 \times 10^{-6}$ \\
\hline
\end{tabular}


(v) The values of $\varepsilon$ in Table 1 lie in the range $m n \leqq \varepsilon \leqq 12 \mathrm{mn}$.

(vi) Even though the relaxation factors $\omega$ in Table 1 are all less than 1, we still (Ref. 18, p. 215) refer to our iterative method as an over-relaxation rather than an under-relaxation method.

The above numerical results indicate that the proposed iterative method is a viable one and, when and are properly chosen, it is competitive with the revised simplex method. These parameters may be chosen experimentally by making a few short test runs starting with $\varepsilon \cong 5 \mathrm{mn}$ and $\omega \cong 0.5$ and picking those values for which $\left\|x^{i+1}-x^{i}\right\|$ approach zero fastest. The main advantages of the method are its robustness, simplicity and ability to handle large problems. 


\section{Acknowledgements}

The author is indebted to his colleaques Dr. Michael Minkoff for his proficient coding of the alqorithm described in this paper and to Kenneth E. Hillstrom for running the test problems. Y. C. Cheng coded an earlier version of the alqorithm. 
Peferences

1. Dantzig, G. B., Linear Programming and Extensions, Princeton University Press, Princeton, liew Jersey, 1963.

2. Murty, K. G., Linear and Combinatorial Programming, John Wiley and Sons, New York, New York, 1976.

3. Fremin, 1. 1., The Relaxation Method of Solving Systems of Inequalities with Convex Functions on the Left Sides, Soviet Mathematics Doklady (transTation), 6, 219-222, 1965.

4. Bregman, L. M., The Method of Successive Projection for Finding a Common Doint of Convex Sets, Soviet Mathematics Doklady (translation), 6, 688-692, 1965.

5. Breqman, L. M., The Relaxation Method of Finding the Common Point of Convex Sets and Its Application to the Solution of Problems in Convex Programming, USSR Computational Mathematics and Mathematical Physics (translation), 7 , $200-217,1967$.

6. Dett7i, W., An Iterative Method, Havinq Linear Rate of Convergence, for Solving a Pair of Dual Linear Programs, Mathematical Programmina, 3 , $302-311,1972$.

7. Polyak, B. T. and Tretiyakov, N. V., Concerning an Iterative Method for Linear Programing and Its Economic Interpretation, Fconomics and Mathematical Methods (Russian), 8, 740-751, 1972.

8. Goffin, J. L., On Converqence Rates of Subgradient Optimization Methods, Mathematical Programming, 13, 329-347, 1977.

9. Mangasarian, 0. L. and Meyer, R. R., Nonlinear Perturbation of Linear Programs, University of Wisconsin, Madison, Wisconsin, Computer Sciences Technical Report No. 326, 1978, to appear in SIAM Journal on Control and Optimization, 17 (6), 1979.

10. Mangasarian, 0. L., Solution of Symmetric Linear Complementarity Problems by Iterative Methods, Journal of Optimization Theory and Applications, 22 , $465-485,1977$.

11. Robinson, S. M., Stability Theory for Systems of Inequalities, Part I: Linear Systems and Part II: Differentiable Nonlinear Systems, SIAM Journal on Numerical Analysis, 12, 754-769, 1975 and 13, 497-513, 1976.

12. Mangasarian, 0. L., Nonlinear Programming, McGraw-Hi11, New York, New York, 1969.

13. Manqasarian, 0. L., Uniqueness of Solution in Linear Programming, Linear Algebra and Its Applications, 25, 151-162, 1979.

14. Golstein, E. G., Private Communication, Moscow, USSR, June 1978. 
15. Fiacco, A. V. and McCormick, G. P., Nonlinear Programming: Sequential Unconstrained Minimization Techniques, John Wiley and Sons, New York, New York, 1968.

16. Bertsekas, D. P., Necessary and Sufficient Conditions for a Penalty Method to be Exact, Mathematical Programming, 9, 87-99, 1975.

17. IMSL Library 1 Reference Manual, Subroutine ZX3LP, Edition 6, International Mathematical and Statistical Libraries, Inc., Houston, Texas, 1977.

18. Ortega, J. M. and Rheinboldt, W. C., Iterative Solution of Nonlinear Equations in Several Variables, Academic Press, New York, New York, 1970. 
Distribution for ANL-79-75

Internal:
W. E. Massey
J. J. More'
R. J. Royston
M. B. Gibson (4)
P. C. Messina
A. B. Krisciunas
M. Minkoff
ANL Contract File
B. S. Garbow
ANL Libraries (5)
H. G. Kaper
TIS Files (6)
G. K. Leaf

External:

DOE-TIC, for distribution per UC-32 (191)

Manager, Chicago Operations and Regional Office, DOE

Chief, Office of Patent Counsel, DOE-CORO

President, Argonne Universities Association

Applied Mathematics Division Review Committee:

P. J. Eberlein, SUNY at Buffalo

G. Estrin, U. California, Los Angeles

W. M. Gentleman, U. Waterloo

J. M. Ortega, North Carolina State U.

E. N. Pinson, Bell Telephone Labs.

S. Rosen, Purdue U.

D. M. Young, Jr., U. Texas, Austin

o. L. Mangasarian, U. Wisconsin, Madison (20) 